\title{
Reviewers and Consultants
}

\section{VOLUME 52, 2008}

The editors and publisher are grateful to the following individuals for their assistance in evaluating manuscripts for publication in the journal. It is only through their efforts that Acta Cytologica is able to maintain the high scientific standards for which it is known.

Fadi W. Abdul-Karim

Pedro P. de Agustín

Måns Åkerman

Mousa A. Al-Abbadi

Patricia Alonso de Ruiz

Carmen Alvarez-Santín

Vamseedhar Annam

Karen M. Atkison

Silvana Audy-Jurkovic

Manon Auger

R. Marshall Austin

Ulrik Baandrup

Christine Bergeron

Aasmund Berner

George G. Birdsong

Paolo Boccato

Miklós Bodó

Renzo Boldorini

Thomas A. Bonfiglio

Mathilde E. Boon

Lukas Bubendorf

Jorge Campos R. de C.

May Chan

Tien-Chun Chang

Liang Cheng

Douglas P. Clark

Terence J. Colgan

Luiz Martins Collaco

Brian T. Collins

William N. Crabtree

Dilip K. Das

Pranab Dey

Daniel C. Dim

Wenancjusz M. Domagala

Antonio Ducrot-Schinini

Jaroslava Dušková

Hormoz Ehya

Marianne Engels

Yener S. Erozan
Canan Ersöz

Guido Fadda

Annabelle Farnsworth

Peter N. G. Fitzgerald

Brendan T. Fitzpatrick

Franco Fulciniti

Hugo Galera-Davidson

Kim R. Geisinger

Murat Gokden

Claude Gompel

Ricardo González-Cámpora

Zenon Gonzalez-Romero

Roberta M. Goodell

Mai Gu

Prabodh K. Gupta

Raj K. Gupta

Xhevdet Harasani

Amanda Herbert

Anders Hjerpe

Yajue Huang

Lucrecia T. Illescas

Teruo Inamoto

Rohit Jain

Gita Jayaram

Matías Jiménez-Ayala

So-Young Jin

William W. Johnston

David B. Kaminsky

Kusum Kapila

Linda Kapusta

Harubumi Kato

Ruth L. Katz

Bryan Knight

Tadao K. Kobayashi

Leopold G. Koss

Helen A. Koutselini

Diane P. Kowalski

Rainer Kraft

Leena Krogerus
Perikala V. Kumar

Sow-Hsong Kuo

Hiroyuki Kuramoto

Gladwyn Leiman

Marianne Lidang

Oscar Lin

Virginia A. LiVolsi

Britt-Marie Ljung

Adhemar Longatto Filho

Usha K. Luthra

Ma Zheng-zhong

Ehud Malberger

Sue Ellen Martin

Shahla Masood

Nicole A. Massoll

Euphemia McGoogan

Ravi Mehrotra

Alexander Meisels

Lilia M. Osorio Mejia de Gomez

Edith Claros Mercado

Pamela M. Michelow

Maria Sultana Mihailovici

Theodore R. Miller

Vatsala Misra

Lopa Modi

Dina R. Mody

Andre L. Moreira

Nicholas J. Mulvany

Zuher M. Naib

Oscar Nappi

Joseph F. Nasuti

Bernard Naylor

Wai-Kuen $\mathrm{Ng}$

Shiro Nozawa

Robert Y. Osamura

Nadir Paksoy

Florence W. Patten

Annette Peck

Raffaele Perrone-Donnorso 
Silvana Pilotti

Rodrigo Prado-Buzeta

Joao Carlos Prolla

Arvind Rajwanshi

Jose Maria Raya

Ibrahim Ramzy

Atsuhiko Sakamoto

Husain Saleh

Mercedes Santamaría Martínez

Yukitoshi Satoh

José P. Schalper

Fernando C. Schmitt

Volker Schneider

Carolina Sforza-Huffman

Naum A. Shapiro

Mark E. Sherman
Azat I. Shibanova

Jan F. Silverman

Jan Siracky

Lambert Skoog

Diane Solomon

Theresa M. Somrak

Radhika Srinivasan

Mark H. Stoler

Kari J. Syrjänen

Aleksander Talerman

Edneia M. Tani

Vinod D. Taylor

Gary M. Tse

Marija Us-Krašovec

László Vass

Alain P. Verhest
Philippe Vielh

G. Peter Vooijs

Neelam Wadhwa

Todd Walker

C. Barry Walsh

Michele M. Weir

Judith A. Whittaker

Helene G. Wiener

David C. Wilbur

Kimie Yamagishi

Grace C. H. Yang

Nancy A. Young

Maureen F. Zakowski

Zhang Ren-yuan 